\title{
Lower thigh-knee lift
}

\section{Eman Yahya Sadek, MD; Khalid Mohamed El-Sherbiny, MD; Ayman A Abdelsalam Elmeligy, MD}

\author{
Department of Plastic and Reconstructive Surgery, Ain Shams University, \\ Cairo, Egypt.
}

\begin{abstract}
Lower thigh laxity is a difficult area to be completely corrected. Although, some techniques may achieve some tightness in this region, skin laxity and ptosis recurs within few months. Thighplasty was performed in 10 patients with skin redundancy and adiposity. Circumferential liposuction was done first. The incision was placed at the posteromedial aspect of the thigh. The medial tissue was pulled upward, posterior and lateral. A posteriorly rotated excision pattern was done. The whole thigh was taut. Skin redundancy at the lower thigh and knee regions was completely eliminated. Follow up ranged between 9-12 months. The described technique is effective in lower thigh lift as well as the whole thigh.
\end{abstract}

\section{Introduction:}

Skin redundancy and adiposity of the thigh may occur at trochanters, medial thigh and or lateral thigh. Medial thigh is more affected than lateral thigh. ${ }^{1,2}$ Anterior thigh laxity is occasionally seen in the massive weight loss (MWL) patient. ${ }^{3}$ Saddle bags and skin redundancy especially at the lower portion are more in MWL patients. Quality of skin and tissue in MWL patients is permanently damaged from being stretched and has inability to maintain tightness or tone. Deformities are divided into two types; deflated and nondeflated. After body recontouring, skin may continue to loosen and sag much faster with age. ${ }^{4}$ Three factors contribute to postoperative skin laxity. First is the diseased skin collagen and elastin. Second is the inability to transmit mechanical forces during surgery; the farther the skin from line of closure, the less effective is the pull. Leaving behind excessively heavy and redundant distal thigh skin adds to the distraction forces. Third, adherence of the skin to underlying fascia prevents tightening beyond the adherence. ${ }^{5}$

The main skin laxity in the medial thigh occurs at the junction of the anterior and medial thigh. Lewis was the first who described medial thigh lift. His technique is posteriorly rotated excision pattern. ${ }^{6}$ Medial thighpalsty techniques were evolved to enhance surgical outcome and minimize complications. They may be considered as effective lifting procedures for upper medial thigh. However they are not effective in the lower medial portion. ${ }^{7-9}$ Also, recurrent ptosis is a possibility. Skin suspension techniques were performed onto the lateral pubis. ${ }^{10-12}$ Results of such techniques were not satisfactory. Lockwood recommended anchoring the subdermal layer of the distal medial thigh tissue to Colles' fascia to allow for a more stable and long-term result. ${ }^{13}$ Vertical incision was reintroduced after especially for post-bariatric patients. ${ }^{14,15}$ Hurwitz described L medial thighplasty in MWL patient. It is composed of upper crescent excision and vertical excision extension. $\mathrm{He}$ deepened the angle between the vertical limb and the upper crescent excision by edging the superior portion of the anterior line posterior. This moves the scar posterior and forms an $\mathrm{L}$ shape. Most of the remaining upper posterior thigh wrinkling could be removed through a triangular infragluteal-posterior extension of the crescent upper medial thigh lift. ${ }^{16}$

Lateral thigh laxity can be corrected with flankoplasty or lower body lift. ${ }^{17-20}$ Tightening was achieved in the upper half more than the lower. Saddle lift described by Ersek helps to lift the buttock and to an extent can stretch 
upper lateral thigh laxity. ${ }^{21}$ Garcia described suction lipectomy and direct excision of skin and fat, with the circular defects being closed with purse-string sutures. ${ }^{22}$ Hurwitz et al. observed that bulge, sag, and loose skin of the lateral thigh reappear within 1 year postoperatively. They described a new positioning to permit an increase in the width of the skin excision and closure under moderate tension. Their technique provides symmetrical correction of the severe saddlebag deformity and better contour to the distal lateral thighs. ${ }^{5}$ Gome et al. described M-shaped thigh lift. The contour is good. Scars are long at the lateral thighs. ${ }^{23}$ Several authors described suspension techniques for lateral thigh lifts in which dermis is sutured to muscular fascia or periosteum to limit scar migration and recurrence of ptosis in this region. ${ }^{24-26}$ Sozer et al. described the spiral lift to correct the guitar deformity. The used incision extends from the inferior crease of the buttocks along the inguinal crease and continues just inferior to the anterior iliac spine, spiralling above the buttocks and meeting the contralateral incision at the sacrum. ${ }^{27}$

Suction-assisted lipectomy is effective especially in lateral and anterior aspects. A bulge around the knee is treated with liposuction. In massive weight loss, liposuction may fail to remodel and tighten the medial thigh where skin laxity can be quite severe and extend down to, and even below the knee. ${ }^{28}$ Aly performed a preliminary liposuction procedure in non-deflated MWL patients to deflate the thighs. Then he did excisional procedures after 6 month. 19

Lower thigh and knee remain troublesome regions for body contouring in patients with generalized lipodystrophy and skin flaccidity. The current study presents a technique that tackles those regions without extension of scar beyond the knee.

\section{Patients and methods:}

10 female patients were presented with skin laxity and adiposity of thighs. Their ages ranged between 25 and 50 years. 5 patients had bariatric surgery. Preoperative examination and standard photography were done. The procedure was done under general anesthesia. Preoperative anticoagulants were used in morbid obesity. There was no need for blood transfusion. Pressure garment was used postoperatively and kept for 6 weeks. Walking was allowed on the next day postoperatively. Patients were discharged after one or two days. Drains were removed when the drain output was less than $50 \mathrm{ml}$. Postoperative follow-up of patients ranged from 9 to 12 months.

\section{Technique:}

Markings are done while the patient is in posterior standing position with the knees apart. Fat deposits are delineated and marked for liposuction Figure(1). The curvilinear incision is marked over the posteromedial aspect of both thighs Figure(2A). The incision starts from the most medial point of the popliteal crease (point a) and passes to the midpoint of the crease (point b). Then it runs upward in a curvilinear manner between medial and posterior compartment and ends at the junction of medial one third and lateral two thirds of the gluteal crease (point c). This incision may be shortened in cases with distal skin redundancy to be at the distal two thirds of the thigh. A horizontal component is added to the vertical incision at the medial third of the infragluteal crease.

The patient is first placed in supine position to perform circumferential liposuction with tumescent technique. Then the patient is turned to prone position to complete liposuction especially the hip and lateral thigh and to perform thighplasty. Curvilinear incision is performed followed by assessing the redundant skin while pulling the skin. Incision is done down to the fascia. Vector of pull is posterior, upward and lateral Figure(2B). Skin excess is excised. Neither dissection nor undermining is performed. Fascia is sutured using 2-0 PDS. Subcutaneous suturing is done using 2-0 Vicryl and intradermal sutures using 2-0 Monocryl. Suction drains are used. Length of the incision and the need to add a horizontal component are determined by the degree and location of thigh laxity. 


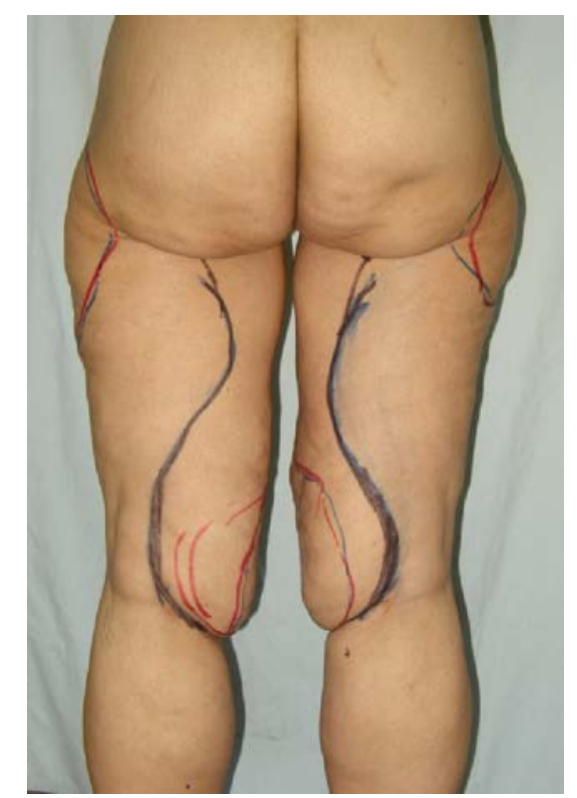

Figure (1): Markings are done while the patient is in posterior standing position.

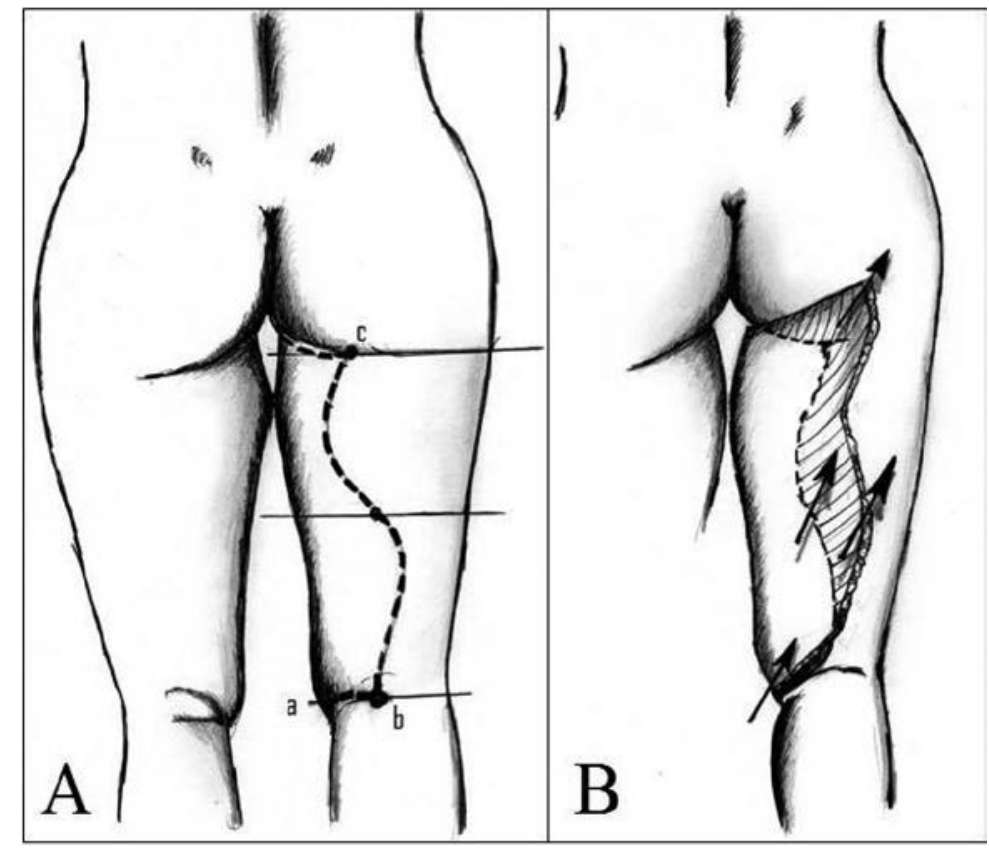

Figure (2): A) Markings of incisions, B) Arrows show vector of pull which is posterior, upward and lateral.

\section{Results:}

Redundant skin of the medial, lateral and anterior thigh was eliminated. Excess skin at lower third of the thigh and knee region is completely eliminated especially medially and posteriorly. Figure(3) shows preoperative and postoperative photographs for a post bariatric patient. Figure(4) shows improvements and changes in knee region. Edema lasted for 3 weeks. There was no hematoma, seroma, or infection. Minimal recurrence occurred in one of post bariatric patients. Other patients were satisfied as regards lifting. Patients were concerned about scar. However, scars fade within 6 to 9 months. Only one patient had hypertrophic scar. 


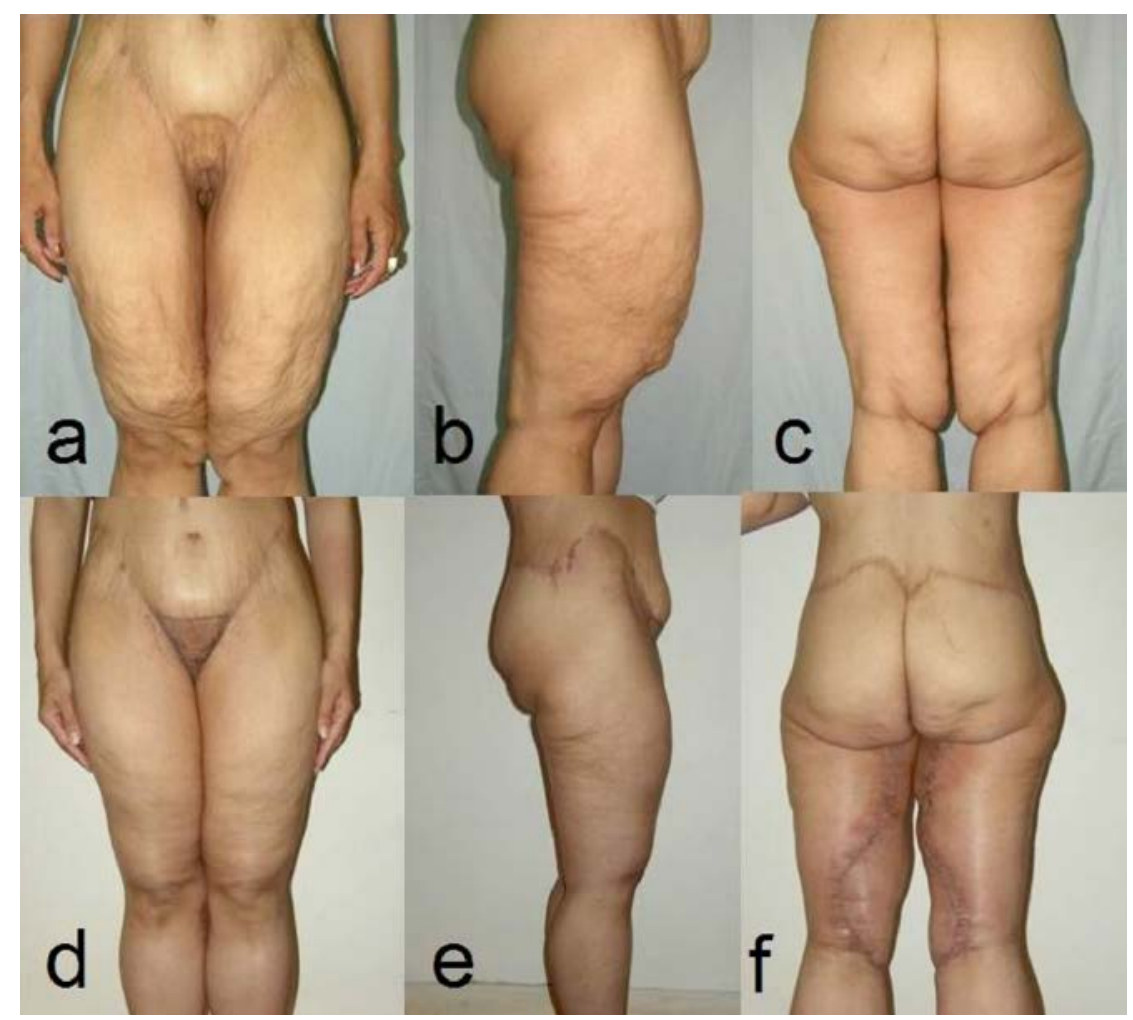

Figure (3): A)Preoperative anterior view, B) Preoperative lateral view, C) Preoperative posterior view, D) Postoperative anterior view, E) Postoperative lateral view, F) Postoperative posterior view.
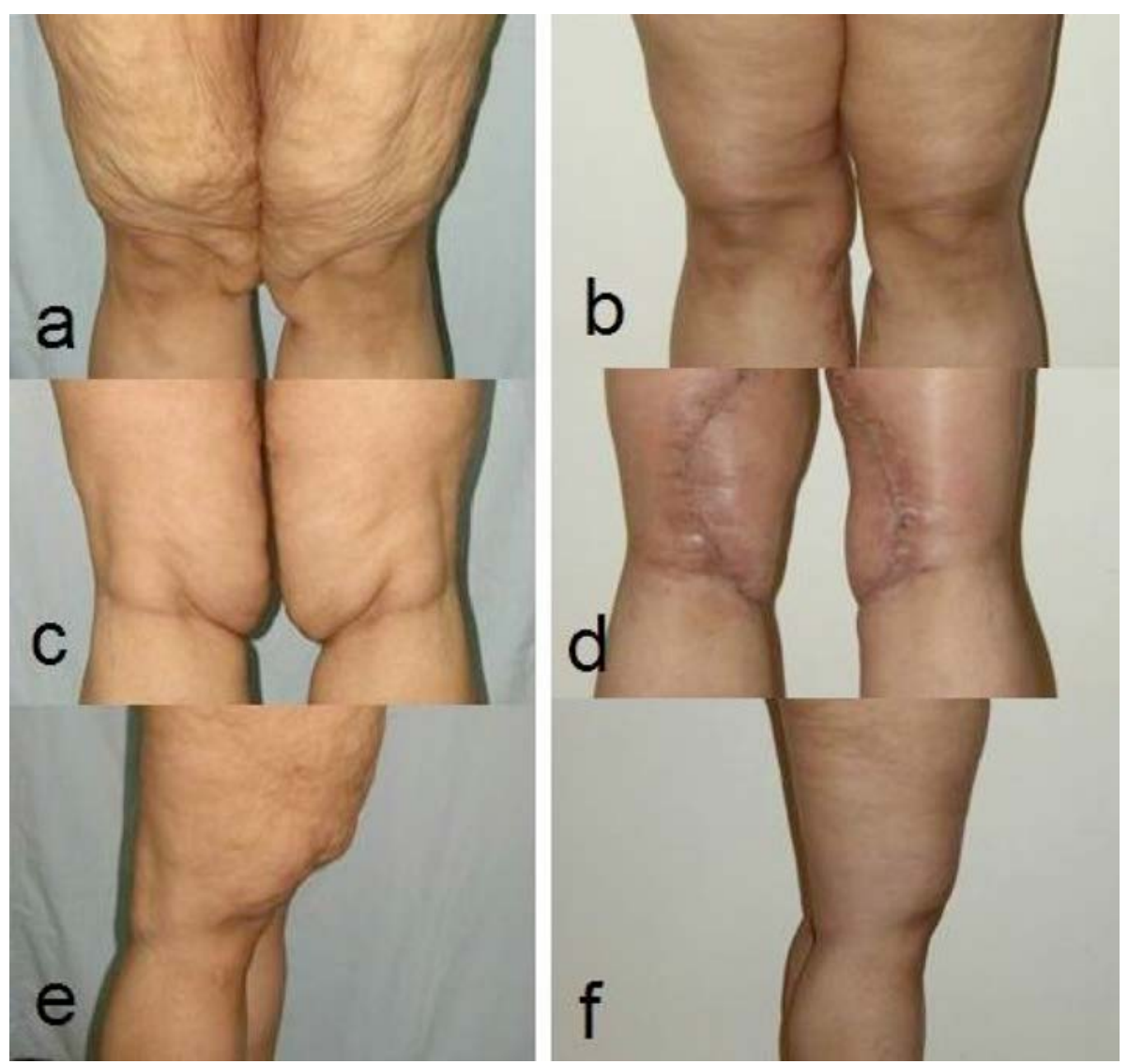

Figure (4): Changes of lower thigh and knee region: A) Preoperative anterior view, B) Postoperative anterior view, C) Preoperative posterior view, D) Postoperative posterior view, E) Preoperative lateral view, F) Postoperative lateral view. 


\section{Discussion:}

Skin redundancy has the potential for skin foldings throughout the thighs in horizontal and vertical axes. The natural cone-shape of the thigh may be turned into log shape. The ideal thigh lift would need to elevate the skin of thigh and tighten the skin in a circumferential manner. Lewis postulated that a complete circumferential incision about the thigh would elevate the skin and subcutaneous tissue whereas long vertical elliptical excision of skin and subcutaneous tissue of the thighs would accomplish the desired tightening. This would make the limb look snug and cone-shape again. ${ }^{6}$ This is impractical as regards blood supply and conspicuous scars. Techniques were innovated to eliminate redundancies, tighten and contour the thigh with the least scars possible. Those techniques were ineffective in lower third and knee region. Medial and lateral suspensions were described to correct ptosis and postpone recurrence as long as possible. Lockwood admitted that SFS suspension may not be effective in heavy thighs. ${ }^{13}$ Mathes and Kenkel stated that in their technique there was no need for tension closure to Colles' fascia. ${ }^{28}$ Results of suspension techniques for lateral thigh lifts were not satisfactory. ${ }^{24-26}$ Hurwitz et al. performed tight approximation of the subcutaneous fascial system. However, they failed to completely correct saddlebag deformity and midthigh laxity in the massive weight loss patient. ${ }^{5}$

Vertical vector approach in medial thigh lift was used by Lockwood. ${ }^{17}$ It may not address many of the anatomic issues encountered in the MWL patient or the entire aging thigh. In concentric medial thigh lift; tightening of the skin of upper medial thigh looks natural because the axes of tractions are perpendicular to the lines of excess skin. ${ }^{9}$ Horizontal vector approach is reconsidered in MWL patients as skin redundancy is more horizontal than vertical. Elabban made vertical ellipse excision. They considered it an easy technique that directly tackles the problem of the redundant skin and fat of the upper inner thighs and alleviates the mechanical friction, and the resultant wound usually heals well with linear scar that is hidden in the inner aspects of the thigh. ${ }^{14}$ Renault's described a vertical extended thighplasty that may extend into the groins with unaccepted scars. ${ }^{15}$ Maths and Kenkle used the vertical vector in nonMWL. They transitioned to a horizontal vector approach in MWL patients. Their technique relies on a vertical incision and horizontal pull. ${ }^{28}$ In L medial thighplasty, the crescent excision corrects upper medial thigh laxity. A vertical extension improves the distal two thirds of oversized thighs and reduces drag on the lateral lift. Some residual laxity recurs, usually in the distal third. Exceptionally loose thighs may need additional resections. ${ }^{16}$ In the current study, horizontal oblique pull was used. This lifted the whole thigh especially the lower third. We did not encounter recurrence of ptosis.

Different incisions were described in thighplasties. Short and hidden scars in the medial buttock crease or in the perineal fold were used. ${ }^{17,9}$ Spiral lift is characterized with long incisions along the inferior gluteal crease and inguinal ligament. ${ }^{27}$ Maths and Kenkle in non-MWL patients performed an incision marked from the level of the ischium along the inner surface of the buttock's fold medially and inferiorly to the labia majora. They added a vertical component if the skin laxity extends beyond the upper one-third of the thigh. In MWL patients, they used a vertical medial incision and added a limited groin incision to the vertical one to remove the dog ear. This has led to a more concealed incision and less undermining of the anterior aspect of the groin. They recommended the use of an additional suprapatellar incision on those patients with significant excess skin over the knee. ${ }^{28}$ Incisions in Lewis technique was mainly posteromedially, vertical and extends along the inguinal ligament. The vertical resection tightens the thigh circumferentially, while, the inguinal incision elevates and snugs the thigh anteriorly. Length of the posteromedial incision varies depending on the degree of thigh ptosis. For milder cases the incision could stop at any point proximal to the knees. ${ }^{6}$ The vertical component of $\mathrm{L}$ medial thighplasty incision ends to be placed posterior. In the current study, our incision is placed at the posteromedial aspect and is formed of three components. First, short lower incision at the 
medial popliteal crease which facilitates lower thigh and knee lift and closed within the vertical one. The upward oblique pull stretches the skin around the knee without the need for extending incision. Second, a curvilinear vertical incision which lies between medial and posterior compartments. It allows posterior, upward and lateral pull. Thus leads to elimination of both horizontal and vertical excess and achievement of better contour. This incision may be shortened in cases with distal skin redundancy to be at the distal two thirds of the thigh. Incision was never extended beyond the knee. Third, a short upper incision at the infragluteal crease medially which facilitates skin redraping and taking off the excess tissue without dog ear or buttock deformities. This scar is concealed in the infragluteal crease. Scar at the posteromedial aspect may not be concealed. However it is not visible during marital intimate relationship. The curvilinear nature of the incision causes no contractures and avoids widened scars. Patients were concerned about scar which faded within 6-9 months. One patient developed hypertrophic scar.

Flankoplasty and lower body lift may tighten the upper lateral thigh. ${ }^{17-20}$ Saddle lift described by Ersek has a mild effect on the upper lateral thigh. ${ }^{21} \mathrm{M}$-shaped thigh lift had a better effect on the expense of long lateral scars. ${ }^{23}$ Hurwitz et al. succeeded with changing intraopearative positioning to achieve better lifting and eliminate severe saddlebag deformity and achieve better contour to the distal lateral thighs. ${ }^{5}$ The spiral lift resulted in improved contour and tension of the medial and lateral thigh. It could not lift the lower thigh effectively. ${ }^{27}$ The current technique could tighten the lateral thigh especially the lower part which may be due to reduction of the whole circumference.

Liposuction is used to recontour the thigh and take off the adiposities. Aggressive liposuction and a vertical medial thighplasty may reduce effect of gravity and postoperative laxity. Circumferential liposuction was performed in this study in all patients. The advantages were elimination of localized adiposities especially around the knee, thigh recontouring and avoidance of dissection and undermining.
Lymphatics can be affected especially in medial thigh lift causing lymphocele, lymphorrhea and may be lymphedema. This complication can be avoided by locating the incision behind the course of the vessels and by leaving a fine layer of hypodermis covering the aponeurosis, possibly containing lymph ducts. ${ }^{29}$ Moreno et al. used lymphsyntigraphy to detect postoperative lymphatic abnormalities after thigh lift. Abnormal lymphoscintigraphy was demonstrated in $30.8 \%$ of patients which results in significant functional and anatomic alterations in the lymphatic system. ${ }^{30}$ Seromas occurs more in $50 \%$ of MWL patients. It is a problem due to large spaces associated with the degree of undermining needed. Seroma incidence could be less with lymphatic preservation, no tension closure and use of drains. ${ }^{31}$ Superfiscial undermining is recommended to decrease lymphatic damage and seroma formation. ${ }^{9,28}$ In this study, we did not encounter lymphatic abnormalities or seroma as we did not do undermining. Circumferential liposuction negates the need for undermining and made lifting easier. Wound dehiscence, scar migration, wide and hypertrophic scar may occur due to closure with tension. We had one hypertrophic scar.

This technique competes the contributing factors of postoperative skin laxity. First, inability to transmit mechanical forces; as the skin is not far from the line of closure and pull is quite efficient. Second, there is no heavy and redundant distal thigh skin left behind which would add to the distraction forces. We had only one post-bariatric patient with minimal postoperative skin laxity.

\section{Conclusion:}

Different techniques of thigh lift could eliminate adiposities and redundancies. Lifting effect was not achieved in all regions of the thigh unless with combined techniques. The lower thigh remained as a difficult region to reach. The Lower thigh-knee lift technique could eliminate redundancies and tighten the whole thigh. It eliminates redundancy at the lower region and knee without extending incision below the knee. Circumferential liposuction contours the thigh and eliminates bulges. Scar at the posteromedial aspect was a concern for patients however it fades within 
few months. This technique is effective in lower thigh and knee regions.

\section{Acknowledgment:}

Authors are grateful for Prof Amr Salah Mahmoud for his guidance and support in the development of this study.

\section{References:}

1- Capella JF: Approach to the lower body after weight loss. In: Aesthetic surgery after massive weight loss. Rubin JP, Matarasso A (Editors); Philadelphia: Elsevier (Publisher); 2007; p. 69-99.

2- David W, Jeffrry M: Current concepts in medial thighplasty. Clinical Plastic Surgery 2008; 35: 151.

3- Aly AS, Capella JF: Staging, reoperation, and treatment of complications after body contouring in the massive-weight-loss patient. In: Reoperative aesthetic and reconstructive plastic surgery. Grotting JC (Editor); St. Louis (MO): Quality Medical Publishing (Publisher); 2nd edition; 2007; p. 1701-1740.

4- Chandawarkar RY: Body contouring following massive weight loss resulting from bariatric surgery. Adv Psychosom Med 2006; 27, 61-72.

5- Hurwitz DJ, Rubin JP, Risin M, Sajjadian A, Sereika S: Correcting the saddlebag deformity in the massive weight loss patient. Plast Reconstr Surg 2004; 114 (5): 1313-1325.

6- Lewis JR: Correction of ptosis of the thighs: The thighs lift. Plast Reconstr Surg 1966; 37: 494.

7- Pitanguy IP: Surgical reduction of the abdomen, thighs and buttocks. Surg Clin North Am 1971; 51(2): 479-489.

8- Vilain R, Dardour JC: Aesthetic surgery of the medial thigh. Ann Plast Surg 1986; 17(3): 176-183, 8.

9- Le Louarn C, Pascal JF: The concentric medial thigh lift. Aesthetic Plast Surg 2004; 28(1): 20-23.

10-Planas J: The Crural Meloplasty For Lifting of the Thighs. Clin Plast Surg 1975; 2: 495.

11-Loeb R: Narrowing of the mons pubis during thigh lifts. Ann Plast Surg 1979; 2: 290.
12-Shaer WD: Gluteal and thigh reduction: Reclassification, critical review and improved technique for primary corrrection. Aesthet Plast Surg 1984; 8: 165.

13-Lockwood TE: Fascial anchoring technique in medial thigh lifts. Plast Reconstr Surg 1988; 82: 299.

14-Ellabban MG, Hart NB: Body contouring by combined abdominoplasty and medial vertical thigh reduction: Experience of 14 cases. Br J Plast Surg 2004; 57: 222-227.

15-Regnault P, Daniel RK: Aesthetic plastic surgery, principles and techniques. Boston, MA: Little Brown and Co. (Publisher); 1984: 655-678 \& 705-720.

16-Hurwitz DJ: Medial thighplasty. Aesth Surg J 2005; 25(2): 180-189.

17-Lockwood TE: Transverse flank-thighbuttock lift with superficial fascial suspension. Plast Reconstr Surg 1991; 87(6): 1019-1027.

18-Lockwood TE: Lower body lift with superficial fascial system suspension. Plast Reconstr Surg 1993; 92(6): 1112-1122.

19-Aly AS: Body contouring after massive weight loss. St. Louis (MO): Quality Medical Publishing (Publisher); 2006.

20-Richter DF, Stoff A, Velasco-Laguardia FJ, Reichenberger MA: Circumferential lower truncal dermatolipectomy. Clin Plastic Surg 2008; 35: 53-71.

21-Ersek RA, Salisbury AV: The saddle lift for tight thighs. Aesth Plast Surg 1995; 19 : 341-343.

22-Garcia JA, Driscoll DN, Donelan MB: Pursestring thigh lift: Direct approach for a problematic deformity. Ann Plast Surg 2006; 57: 330.

23-Gomes HC, Ribeiro RB, Bizerra MM, Lucca AF, Ferreira LM: The M-shaped thigh lift. Plast Reconstr Surg 2008; 122(2):100e-101e.

24-Agris J: Use of dermal fat suspension flaps for thigh and buttocks lifts. Plast Reconstr Surg 1977; 59: 817.

25-Teimourian B, Adham MN: Anterior periosteal dermal suspension with suction curettage for lateral thigh lipectomy. Aesthet Plast Surg 1982; 6: 207.

26-Gurrerosantos J: Secondary hip-buttockthighplasty. Clin Plast Surg 1984; 11: 491. 
27-Sozer SO, Agullo FJ, Palladino H: Spiral lift: Medial and lateral thigh lift with buttock lift and augmentation. Aesth Plast Surg 2008; 32: 120-125.

28-Mathes DW, Kenkel JM: Current concepts in medial thighplasty. Clin Plast Surg 2008; 35(1): 151-163.

29-Leitner DW, Sherwood RC: Inguinal lymphocele as a complication of thighplasty. Plast Reconstr Surg 1983; 72:878-881.ep;18(9):1160-1164.
30-Moreno CH, Neto HJ, Junior AH, Malheiros CA: Thighplasty after bariatric surgery: Evaluation of lymphatic drainage in lower extremities. Obes Surg 2008; S. 31-Kenkel JM: Safety Considerations and avoiding complications in the massive weight loss patient. Plast Reconst Surg 2006; 117 (1S), 74-86. 